\title{
Brain metastases from lung cancer responding to erlotinib: the importance of EGFR mutation
}

\author{
R. Porta, J.M. Sánchez-Torres, L. Paz-Ares, B. Massutí, N. Reguart, C. Mayo, \\ P. Lianes, C. Queralt, V. Guillem, P. Salinas, S. Catot, D. Isla, A. Pradas, A. Gúrpide, \\ J. de Castro, E. Polo, T. Puig, M. Tarón, R. Colomer and R. Rosell
}

ABSTRACT: Median survival of patients with brain metastases from nonsmall cell lung cancer (NSCLC) is poor and more effective treatments are urgently needed. We have evaluated the efficacy of erlotinib in this setting and its association with activating mutations in the epidermal growth factor receptor (EGFR) gene.

We retrospectively identified patients with NSCLC and brain metastases treated with erlotinib. EGFR mutations in exons 19 and 21 were analysed by direct sequencing. Efficacy and tolerability were compared according to EGFR mutational status.

69 NSCLC patients with brain metastases were identified, 17 of whom harboured EGFR mutations. Objective response rate in patients with EGFR mutations was $82.4 \%$; no responses were observed in unselected patients $(p<0.001)$. Median $(95 \% \mathrm{Cl})$ time to progression within the brain for patients harbouring EGFR mutations was 11.7 (7.9-15.5) months, compared to $5.8(5.2-$ 6.4) months for control patients whose EGFR mutational status had not been assessed $(p<0.05)$. Overall survival was $12.9(6.2-19.7)$ months and $3.1(2.5-3.9)$ months $(p<0.001)$, respectively. The toxicity of erlotinib was as expected and no differences between cohorts were observed.

Erlotinib is active in brain metastases from NSCLC; this clinical benefit is related to the presence of activating mutations in exons 19 or 21 of the EGFR gene.

KEYWORDS: EGFR, metastases, mutation screening, nonsmall cell lung cancer, targeted therapy

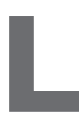

ung cancer is the leading cause of cancerrelated death worldwide. Brain metastases from nonsmall cell lung cancer (NSCLC) are present in $20-30 \%$ of patients [1] and are associated with a poor prognosis despite treatment with whole brain radiotherapy (WBRT), with a median survival of $<6$ months [2]. Apart from WBRT, few treatment options are currently available for these patients.

Tyrosine kinase inhibitors (TKIs) of the epidermal growth factor receptor (EGFR) are novel treatment options for advanced NSCLC, with a reported response rate of $9 \%$ in an unselected nonchemotherapy-naïve population [3]. Activating EGFR mutations within the tyrosine kinase (TK) domain are found to be highly associated with sensitivity to the EGFR TKIs gefitinib or erlotinib in advanced NSCLC [4-6]. Almost $90 \%$ of all known mutations in the TK domain of the EGFR gene are located in exon 19 (in-frame deletion of the conserved sequence LREA) or in exon 21 (L858R point mutation). Recent studies have shown that these EGFR mutations are highly oncogenic in transgenic mice and maintenance of the lung tumours in these mice is dependent on continued expression of the EGFR mutants $[7,8]$. These data suggest that NSCLC expressing EGFR mutants is itself a different molecular entity $[9,10]$.

Although individual case reports of patients achieving objective responses to erlotinib or gefitinib have been published, the role of TKIs in patients with brain metastases remains unclear. To address this issue, we retrospectively evaluated the efficacy of erlotinib in a series of patients with brain metastases from NSCLC and its association with the presence of activating mutations in the EGFR gene. Safety was evaluated as a part of the analysis.
AFFILIATIONS

A full list of the authors and their affiliations can be found in the Acknowledgements section.

\section{CORRESPONDENCE}

R. Colomer

M.D. Anderson España Cancer Center C/Arturo Soria 270 28033 Madrid

Spain

E-mail: rcolomer@seom.org

Received:

Dec 112009

Accepted after revision:

June 212010

First published online:

July 012010 


\section{MATERIALS AND METHODS Patients}

We retrospectively evaluated patients with NSCLC and metastatic dissemination to the brain, who had been registered in the Spanish Lung Adenocarcinoma Data Base Study (SLADB) from April 2005 to May 2006. The SLADB is a large database sponsored by the Spanish Lung Cancer Group (SLCG), whose aim was to evaluate the feasibility of largescale screening for EGFR mutations in NSCLC patients and to examine the association between the mutations and the outcomes of the treatment with erlotinib. Primary tumour biopsy specimens from 2,105 NSCLC patients were analysed [11] and only those harbouring EGFR mutations were included in the database.

In addition, in order to have a control population of patients with brain metastasis from lung cancer, we consulted the TargeT study database and picked patients with brain metastasis enrolled during the same time period whose EGFR mutational status was either unknown or wild-type. The TargeT study is a Spanish nonrandomised phase II trial evaluating the efficacy and safety of first- and second-line erlotinib in patients with histologically confirmed stage IIIB or IV NSCLC. Erlotinib was given at a daily dose of $150 \mathrm{mg}$ until disease progression or severe toxicity.

Both the SLADB and the TargeT study were approved by the corresponding institutional review boards and patients provided written informed consent prior to enrolment.

\section{Efficacy and safety}

Assessment of treatment efficacy at the brain level was periodically performed by brain magnetic resonance imaging or computed tomography (CT) scan, according to the clinical practice of each site. Lung tumour response was evaluated by CT scan. Liver or bone metastases, if present, were evaluated by upper abdominal CT scan and bone scan, respectively. Efficacy is reported in terms of objective response rate according to the Response Evaluation Criteria in Solid Tumours [12], time to progression (TTP) and overall survival (OS). TTP of the intracranial lesions was measured from the date of first erlotinib intake until the date of progression within the brain or last follow-up. OS was measured from the date of first erlotinib intake until death or last survival follow-up. Safety data consists of the adverse events related to erlotinib according to the National Cancer Common Toxicity Criteria version 3 grading system [13].

\section{EGFR mutation analysis}

The analysis of EGFR mutations was performed at the central laboratory of the SLCG at the Catalan Institute of Oncology (Hospital Germans Trias i Pujol, Badalona, Spain). EGFR mutations in exons 19 and 21 were analysed as described previously [11]. For more details on genetic analysis see the online supplementary data S1.

\section{Statistical analysis}

Patient characteristics are listed by their frequencies for qualitative variables and by median values and ranges for quantitative variables. Differences among response rates were analysed by the Chi-squared test or Fisher's exact test, as appropriate. Actuarial progression and survival curves were generated using the Kaplan-Meier method. The log-rank test was used to detect differences between subgroups. Two-sided p-values $<0.05$ were considered statistically significant. Statistical analyses were performed using SPSS for Windows version 13.0 (SPSS, Inc., Chicago, IL, USA).

\section{RESULTS}

\section{Patient and tumour characteristics}

This retrospective analysis includes 69 patients with NSCLC metastatic to the brain, whose main baseline and clinical characteristics are summarised in table 1 . Most of the patients were current or former smokers (68.0\%), with adenocarcinoma $(68.0 \%)$ and an Eastern Cooperative Oncology Group performance status of one $(61.5 \%) .37(53.6 \%)$ patients were male.

Of the 69 patients with brain metastases, 17 (24.6\%) harboured mutations in the EGFR gene. An in-frame deletion in exon 19 (E746-A750) was found in $12(70.6 \%)$ patients, while a point mutation in exon 21 (L858R) was detected in the remaining five (29.4\%) patients. The majority of patients with EGFR mutations were female $(64.7 \%)$, never-smokers $(64.7 \%)$ and had adenocarcinomas $(82.4 \%)$.

In contrast, the 52 control cases $(75.4 \%$ of the whole series) from the TargeT study were unselected patients, whose EGFR mutational status had not been assessed (50 patients) or had confirmed wild-type EGFR gene (two cases); these control patients were mainly males $(59.6 \%)$ and former or current smokers $(78.8 \%)$; adenocarcinoma was also the predominant histology in this group $(63.5 \%)$.

Of the entire series, 55 patients were treated with standard WBRT prior to erlotinib treatment: nine (16.4\%) patients with EGFR mutation and $46(84.6 \%)$ in the control group. Approximately half $(47.1 \%)$ of the patients with EGFR mutations did not receive WBRT, and oral erlotinib was the sole treatment. In contrast, all control patients with available data of treatment had received erlotinib plus radiotherapy. Median (range) time from the end of WBRT treatment until the beginning of erlotinib intake was 42 (9-270) days. None of the patients received stereotactic radiation or underwent resection of the brain lesions.

Nine $(52.9 \%)$ of the 17 patients harbouring EGFR mutations and $23(44.2 \%)$ control cases received chemotherapy after erlotinib treatment failure.

\section{Treatment efficacy}

Response was not evaluable in 16 patients due to early death; 53 patients were evaluable for response. $14(26.4 \%)$ patients attained an objective response in the brain lesions. All of them harboured mutations in the EGFR gene. Three patients with EGFR mutations had stabilisation of the intracranial lesions. Therefore, the objective response rate in the subgroup of evaluable patients with EGFR mutations was $82.4 \%$, with complete resolution of the brain metastases in eight cases $(47.1 \%)$ and partial response in six (35.3\%).

No objective response within the brain was reported among patients in the control cohort, even though they had all received WBRT. Difference in response rate between patients with EGFR mutations and unselected control patients was statistically significant ( $p<0.001$; table 2 ). Remarkably, however, $77.8 \%$ of 


\begin{tabular}{|c|c|c|c|}
\hline Characteristics & All patients & $\begin{array}{c}\text { Cases with EGFR } \\
\text { mutations }\end{array}$ & Control cases \\
\hline Subjects & $69(100)$ & $17(24.6)$ & $52(75.4)$ \\
\hline \multicolumn{4}{|l|}{ Sex } \\
\hline Male & $37(53.6)$ & $6(35.3)$ & $31(59.6)$ \\
\hline Age yrs & $55(26-81)$ & $56(26-70)$ & 55 (39-81) \\
\hline \multicolumn{4}{|l|}{ Smoking history } \\
\hline Never-smokers & $22(32.0)$ & $11(64.7)$ & $11(21.2)$ \\
\hline Former or current smokers & $47(68.0)$ & $6(35.3)$ & $41(78.8)$ \\
\hline \multicolumn{4}{|l|}{ Histology } \\
\hline Adenocarcinoma & $47(68.0)$ & $14(82.4)$ & $33(63.5)$ \\
\hline 0 & $9(13.0)$ & $1(5.8)$ & $8(16.3)$ \\
\hline 1 & $40(58.0)$ & $10(58.8)$ & $30(61.2)$ \\
\hline 2 & $14(20.2)$ & $5(29.4)$ & $9(18.4)$ \\
\hline 3 & $2(2.9)$ & 0 & $2(4.1)$ \\
\hline Unknown & $4(5.8)$ & $1(5.8)$ & $3(5.7)$ \\
\hline \multicolumn{4}{|l|}{ Erlotinib treatment line } \\
\hline $1^{\text {st }}$ & $26(37.7)$ & $10(58.8)$ & $16(30.8)$ \\
\hline $2^{\text {nd }}$ & $20(29.0)$ & $5(29.4)$ & $15(28.8)$ \\
\hline $3^{\text {rd }}$ & $23(33.3)$ & $2(11.8)$ & $21(40.4)$ \\
\hline \multicolumn{4}{|l|}{ Extracranial metastases } \\
\hline Yes & $45(65.2)$ & $10(58.8)$ & $35(67.3)$ \\
\hline No & $37(53.6)$ & $8(47.1)$ & $29(55.8)$ \\
\hline \multicolumn{4}{|l|}{ EGFR mutation } \\
\hline Exon 19 deletion & $12(17.4)$ & $12(70.6)$ & \\
\hline Exon 21 L858R & $5(7.2)$ & $5(29.4)$ & \\
\hline
\end{tabular}

Data are presented as n (\%) or median (range). Data for the entire series, for those patients harbouring an EGFR gene mutation and for control cases are shown. EGFR: epidermal growth factor receptor; ECOG PS: Eastern Cooperative Oncology Group performance status; WBRT: whole brain radiotherapy.

patients with the unknown EGFR mutational status showed stabilisation of the brain disease after treatment with WBRT plus erlotinib.

In the subgroup of patients with EGFR mutations, eight (47\%) patients did not receive WBRT and erlotinib was the only treatment; of those, six (75\%) achieved an objective response (complete response and partial response) (table 3). A representative case of brain response to erlotinib (case number 5) is shown in figure 1 . All patients but one receiving erlotinib plus WBRT showed response of the intracranial disease (table 3).

In addition to the efficacy within the brain, the response of the primary tumour and extracranial metastases (if present) was also evaluated in the subgroup of patients with activating
EGFR mutations (table 3). All patients with EGFR mutations showed tumour response or disease stabilisation. All patients but one achieving an objective response of the intracranial lesions also attained a response in the extracranial locations. Two of the three patients with stable disease in the brain attained a partial response in the primary tumour as well as in the extracranial metastases. One patient had stable disease at both the thoracic and brain levels.

Median (95\% CI) time to progression in the brain for the entire series was 2.9 (2.3-3.5) months. Patients harbouring EGFR mutations had a median (95\% CI) TTP within the brain of 11.7 (7.9-15.5) months, compared to 5.8 (5.2-6.4) months in the control cohort $(\mathrm{p}<0.05)$ (fig. $2 \mathrm{a})$. Of the 13 progressing patients harbouring EGFR mutations, six experienced disease progression 


\begin{tabular}{lccc} 
TABLE 2 & $\begin{array}{c}\text { Response of brain metastases in patients treated } \\
\text { with erlotinib }\end{array}$ \\
All patients & $\begin{array}{c}\text { Cases with } \\
\text { EGFR mutations }\end{array}$ & Control cases \\
\hline Patients n & 53 & 17 & 36 \\
CR & $8(15.1)$ & $8(47.1)$ & 0 \\
PR & $6(11.3)$ & $6(35.3)$ & 0 \\
CR+PR & $14(26.4)$ & $14(82.4)$ & 0 \\
SD & $31(58.5)$ & $3(7.6)$ & $28(77.8)$ \\
PD & $8(15.3)$ & 0 & $8(22.2)$ \\
\hline & & & \\
Data are presented as n (\%), unless otherwise stated. Data for the entire series, \\
for those patients harbouring EGFR gene mutations and for control cases are \\
shown. EGFR: epidermal growth factor receptor; CR: complete response; PR: \\
partial response; SD: stable disease; PD: progressive disease.
\end{tabular}

in the primary lung lesions, four within the brain and three in the liver (table S1 in the online supplementary material).

Median (95\% CI) OS for the entire population was 4.3 (2.36.2) months. Patients harbouring EGFR mutations had a median OS of 12.9 (6.2-19.7) months while the control group showed a median (95\% CI) OS of 3.1 (2.5-3.9) months $(\mathrm{p}<0.001)$ (fig. 2b). 1-yr survival was $69 \%$ in those patients with mutations and $9 \%$ in the unselected population $(\mathrm{p}<0.001)$ (table S2 in the online supplementary material).

No differences in response rate, TTP within the brain and OS were found according to performance status and treatment line (data not shown) among patients harbouring EGFR mutations, but the small population does not allow definitive conclusions.

\section{Treatment toxicity}

The most common side-effects of erlotinib were rash and diarrhoea. Skin disorders occurred in 37 (53.6\%) cases. Grade $\geqslant 3$ skin toxicity, including desquamative lesions, pruritus, acne, conjunctivitis and alopecia were more frequent in patients with EGFR mutations (18.7\%) than in the control cases $(11.5 \%)$, although this difference did not reach statistical significance $(p=0.17)$. The initial dose of erlotinib was reduced to $100 \mathrm{mg}$ a day in five patients with grade 3 skin toxicity. This measure was sufficient to decrease the skin toxicity to grade 2 . Gastrointestinal toxicity was mild. 17 (25\%) of the 69 patients experienced some gastrointestinal symptom. Grade 3-4 diarrhoea was reported in $4 \%$ of patients in the control group, whereas none of the patients with EGFR mutations developed severe diarrhoea.

\section{DISCUSSION}

This retrospective study shows that the EGFR TKI erlotinib is active in patients with brain metastases from NSCLC. We have observed an overall response rate of $26.4 \%$ in a series of 69 NSCLC patients with metastatic dissemination to the brain treated either with WBRT plus erlotinib or erlotinib alone. Disease control was achieved in an impressive $84.9 \%$ of the patients. We have also identified a group of patients with brain metastases in whom erlotinib is particularly active. Those patients harbouring activating mutations in the EGFR TK domain attained an objective response rate of $82.4 \%$, in some cases with highly dramatic complete responses $(47.1 \%)$. In contrast, unselected patients, whose EGFR mutational status

TABLE 3 Tumour response by site among patients harbouring EGFR mutations

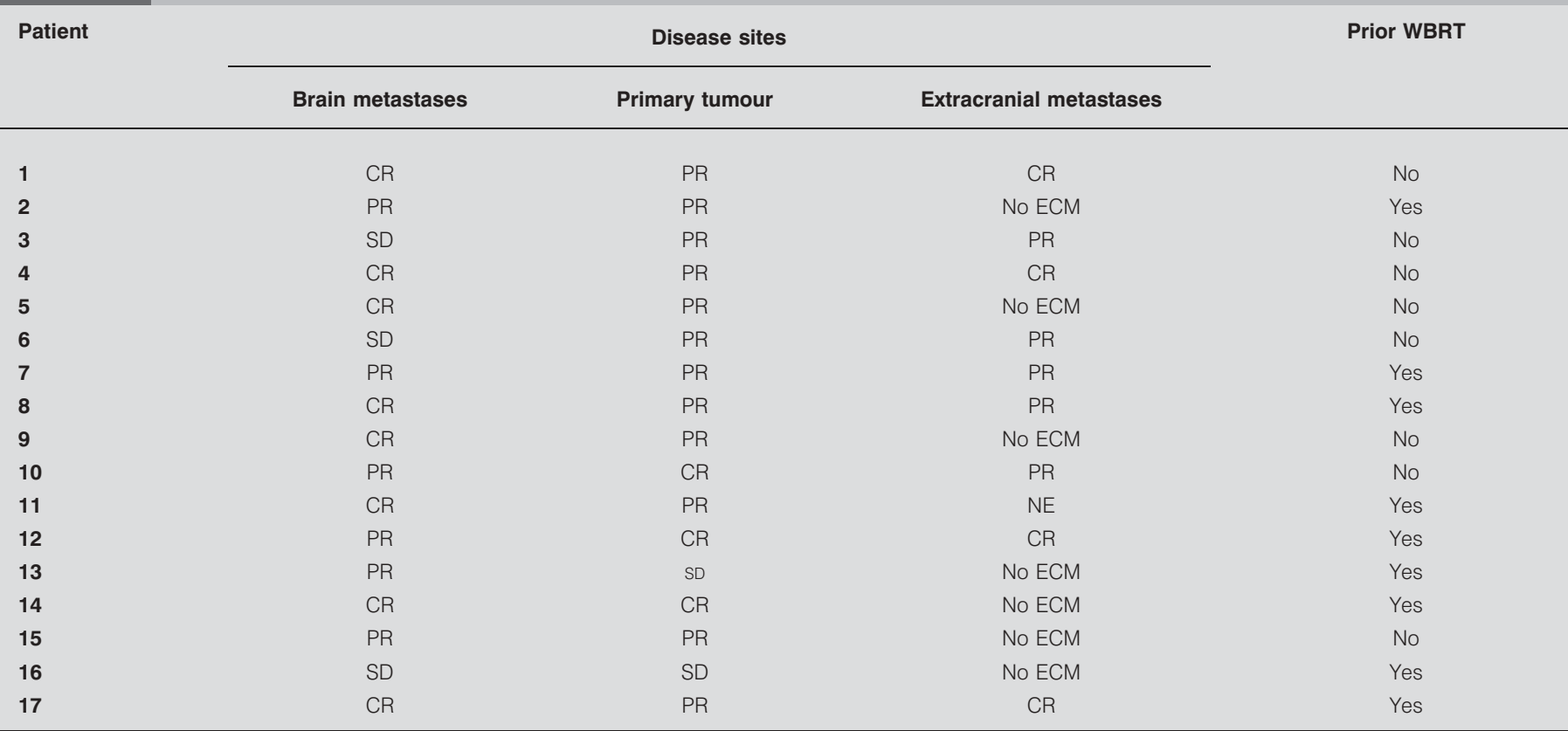

Data of response to treatment of the primary lung tumour, brain metastasis and extracranial metastases are shown. Whole brain radiotherapy (WBRT) is listed for each patient. EGFR: epidermal growth factor receptor; CR: complete response; PR: partial response; NE: not evaluable; SD: stable disease; ECM: extracranial metastases. 

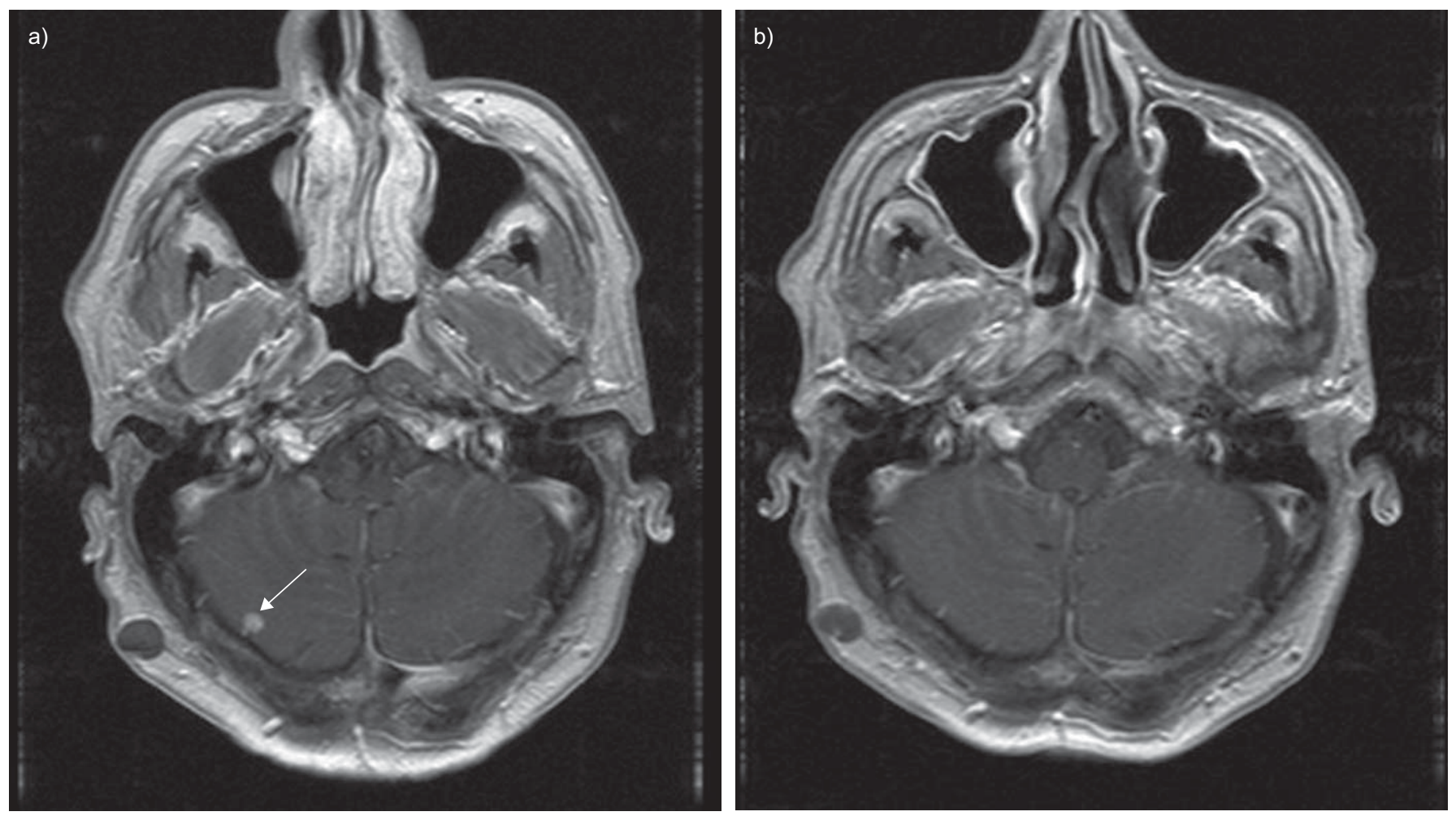

FIGURE 1. Brain magnetic resonance imaging. Axial T1-weighted image after contrast (Gd) administration of patient 5 , a male with adenocarcinoma of the lung and a brain metastasis, harbouring exon 19 deletion. a) A brain metastasis in the right hemisphere of the cerebellum before initiating erlotinib (white arrow). A sebaceous cyst is seen into the right subcutaneous tissue. b) Complete response of the cerebellum metastasis after 4 months of treatment with erlotinib.

was either unknown or wild-type, showed no objective responses, even though disease control occurred in $77.8 \%$ of the population. A significant difference in TTP within the brain lesions and in OS was also observed according to EGFR mutational status. TTP within the brain for patients harbouring EGFR mutations (11.7 months) was twice that for unselected patients (5.8 months). Furthermore, patients harbouring EGFR mutations had four-fold longer OS (12.9 months) than those patients in the control group (3.1 months). 1-yr survival (69\%) for patients with mutations was particularly remarkable, since median OS in unselected patients with lung cancer metastatic to the brain is normally $<6$ months after conventional therapy [2]. Median TTP for patients with the mutated EGFR gene was of similar magnitude to that described in larger series with erlotinib. In a recent prospective study with erlotinib, reported median progression-free survival was 14 months [11], and in a pooled analysis examining five studies of first-line treatment with erlotinib or gefitinib in patients in whom EGFR mutational status was analysed, median progression-free survival for those patients harbouring activating mutations was 11.8 months [14]. By contrast, median OS in our series was shorter than that reported by other authors. This result could be partly due to a shorter follow-up in our study, but it also may reflect the worse prognosis of those patients with brain metastasis and the modest results yielded by other therapeutic approaches, thus underlining the benefit provided by erlotinib. In the work from RoselL and co-workers $[10,11]$ treatment with erlotinib reached a median $(95 \% \mathrm{CI})$ progression-free survival of 14 (11.3-16.7) months for patients without brain metastases and 10 (5.6-14.4) months for those with brain metastases $(\mathrm{p}=0.31)$. Median $(95 \% \mathrm{CI})$ survival was 28 (21.534.4) months for patients without brain metastases and 18 (431.9) months for patients with brain metastases $(p=0.008)$ (see appendix in the online supplementary material) [11].

Several reports support that stereotactic radiosurgery, Gamma Knife or linear accelerator, with or without WBRT, are interesting local therapeutic approaches for a limited number of small brain metastases and good prognosis. However, most cases require a systemic approach to provide a treatment for the extracranial disease [15]. It has been suggested that EGFR mutations confer radiosensitivity in vitro [16], and recently Gow et al. [17] have concluded that the presence of EGFR mutations is an independent predictor of response to WBRT in brain metastases of lung adenocarcinoma. The impact of erlotinib on brain metastases might thus have been masked by the effects of radiation therapy to the brain. However, our study clearly shows that those patients with brain metastases and EGFR mutations are better responders to erlotinib, whether or not they had received previous WBRT. All patients with EGFR mutations obtained benefit within the brain $(82.4 \%$ with objective response and $7.6 \%$ with stable disease as the best response), and $47.1 \%$ attained a complete remission of the cerebral lesions. Interestingly, six (42.9\%) of the 14 patients with EGFR mutations achieving objective tumour response had not received brain radiation therapy, and four of these six attained a complete remission of brain lesions. This finding strongly supports the role of erlotinib in the response of the brain metastases. 

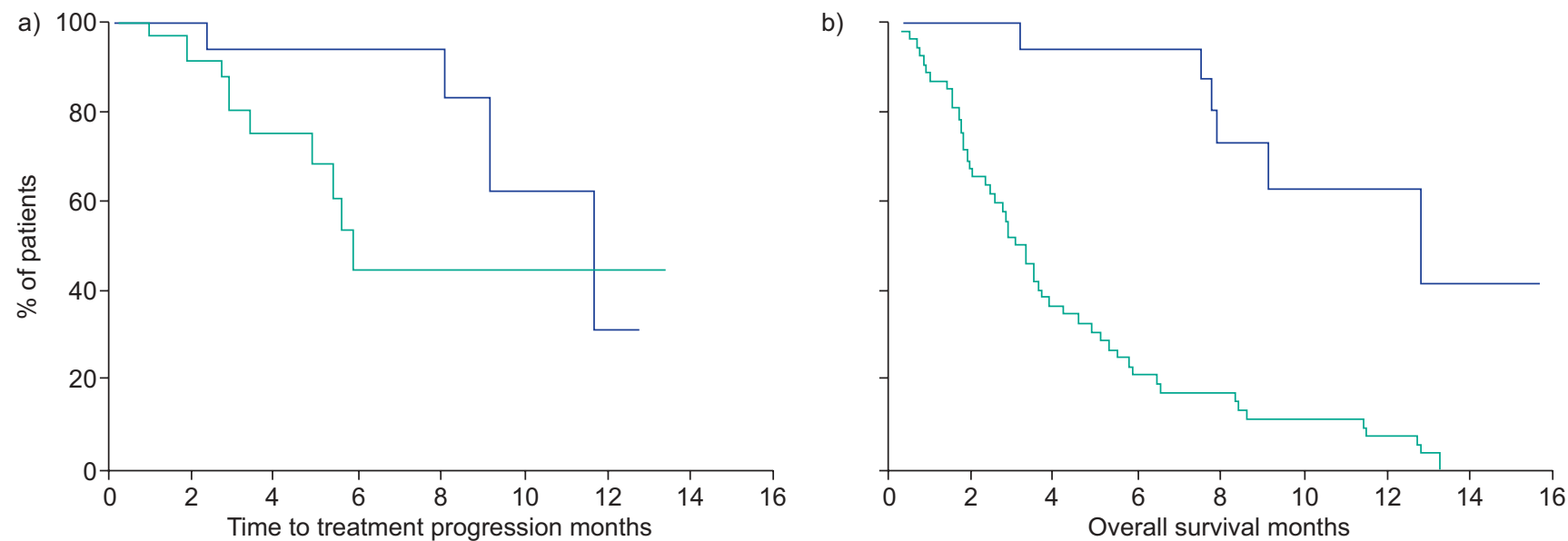

FIGURE 2. a) Median time to progression (TTP) in the brain. Patients harbouring EGFR mutations (blue line) had a median (95\% Cl) TTP within the brain of 11.7 (7.915.5) months, compared to 5.8 (5.2-6.4) months in the control cohort (green line) $(p<0.05)$. b) Median overall survival (OS). Patients harbouring EGFR mutations (blue line) had a median $(95 \% \mathrm{Cl})$ OS of $12.9(6.2-19.7)$ months while the control group (green line) showed a median OS of $3.1(2.5-3.9)$ months ( $<<0.001)$. In the control groups the EGFR mutational status was either unknown or wild-type.

Moreover, the efficacy of erlotinib in brain metastases was paralleled by its efficacy in the lung primary lesions and in other metastatic sites. All patients with EGFR mutations responding to treatment within the brain also responded in the extracranial lesions. In fact, brain lesions seem to be more sensitive to erlotinib than thoracic tumours: eight patients with complete responses within the brain, four of whom were treated only with erlotinib, attained partial responses in their primary tumours. Therefore, we can conclude that erlotinib is active both in brain metastases and in lung primary lesions and other metastatic sites more accessible than the brain.

In the present study, there was a difference in the number of treatment lines between patients with EGFR mutations and patients with unknown EGFR mutational status; unselected patients were more likely to have received previous therapies. While this could account for differences in outcomes between the two groups of patients, $41.2 \%$ of patients with EGFR mutations received erlotinib as a second or further line of treatment, and median TTP in this subgroup remained longer than 11 months. Moreover, among patients harbouring EGFR mutations, no significant differences in response rate, TTP within the brain and OS were detected according to line of treatment and performance status, but these data should be cautiously interpreted due to the small size of the subgroups.

Our findings support the hypothesis that erlotinib is able to cross the blood-brain barrier and displays efficacy against intracranial metastasis. In the past, the response of malignancies involving the brain has been anecdotal [18], which might reflect the absence of active medical treatments, rather than the refractoriness of brain lesions to all forms of therapy. We have previously reported that tamoxifen, which is usually regarded as ineffective in breast cancer involving the brain, induced a complete response in a patient with brain metastases from breast cancer [19].

The results observed in the present series of patients with brain metastases confirm other isolated reports of the efficacy of EGFR TKIs [20-24]. Gefitinib has been reported to be active in a series of patients with brain metastases [21-24], most of them Asiatic, although a high incidence of recurrence at the brain level after treatment with gefitinib has also been addressed [25]. In a prospective trial, CERESOLI et al. [24] showed efficacy of gefitinib on brain metastases from 41 patients with NSCLC, with a median overall survival of 5 months. None of the mentioned studies selected the patients for treatment according to the mutational status of the EGFR gene, or carried out this analysis. It has been pointed out that gefitinib may have an incomplete penetration though the blood-brain barrier [26] and its effectiveness for the treatment of brain metastasis may depend on the disruption of the barrier [27].

Finally, the tolerability of oral TKIs in patients with brain metastases has not been specifically addressed before, although this is particularly relevant in the case of oral drugs. Erlotinib was well tolerated overall in patients with brain metastases, with skin toxicity and diarrhoea as the most common adverse events. Skin toxicity has been associated with clinical benefit to erlotinib, but its relationship with EGFR mutations has not been evaluated [28]. In the present study, a nonsignificant trend towards more severe skin toxicity in patients with EGFR mutations was observed.

In conclusion, erlotinib is well tolerated and active against brain metastases in NSCLC patients. The routine assessment of EGFR mutations in NSCLC patients with intracranial lesions is warranted.

\section{SUPPORT STATEMENT}

Financial support was provided by the Susan G. Komen Breast Cancer Foundation (PDF 0504073) (R. Porta.) and the Spanish Society of Medical Oncology, Research Projects 2005 (R. Porta). The Lung Adenocarcinoma Data Base Study (SLADB) is sponsored by the Spanish Lung Cancer Group and the TargeT trial is sponsored by Roche Farma, S.A. (Spain).

\section{STATEMENT OF INTEREST}

A statement of interest for the present study can be found at www.erj. ersjournals.com/site/misc/statements.xhtml 


\section{ACKNOWLEDGEMENTS}

The author affiliation details are as follows: R. Porta, Dept of Medical Oncology, Catalan Institute of Oncology, Hospital Universitari Dr. Josep Trueta, Girona and Institut de Recerca Biomèdica de Girona (IdIBGi), Hospital Universitario Dr. Josep Trueta, Girona; J.M. Sánchez, Dept of Medical Oncology, M.D. Anderson España Cancer Center, Madrid; L. Paz-Ares, Dept of Medical Oncology, Hospital Universitario Virgen del Rocío, Seville; B. Massutí, Dept of Medical Oncology, Hospital General Universitario de Alicante, Alicante; N. Reguart, Dept of Medical Oncology, CSC-Hospital Clinic de Barcelona, Barcelona; C. Mayo, Pangaea Biotech, USP Institut Universitari Dexeus, Barcelona; P. Lianes, Dept of Medical Oncology, Hospital de Mataró, Mataró; C. Queralt, Pangaea Biotech, USP Institut Universitari Dexeus, Barcelona and Dept of Medical Oncology, Catalan Institute of Oncology and Autonomous University of Barcelona, Hospital Germans Trias i Pujol, Barcelona; V. Guillem, Dept of Medical Oncology, Institut Valencià d'Oncologia - IVO, Valencia; P. Salinas, Dept of Medical Oncology, Hospital Sanitas La Zarzuela, Aravaca; S. Catot, Dept of Medical Oncology, Althaia-Xarxa Assistencial de Manresa, Manresa; D. Isla, Dept of Medical Oncology, Hospital Clínico Universitario Lozano Blesa, Zaragoza; A. Pradas, Dept of Medical Oncology, Catalan Institute of Oncology and Autonomous University of Barcelona, Hospital Germans Trias i Pujol, Barcelona; A. Gúrpide, Dept of Medical Oncology, Clínica Universitaria de Navarra, Pamplona; J. de Castro, Dept of Medical Oncology, Hospital Universitario La Paz, Madrid; E. Polo, Dept of Medical Oncology, Hospital Ernest Lluch, Calatayud; T. Puig, Institut de Recerca Biomèdica de Girona (IdIBGi), Hospital Universitario Dr. Josep Trueta, Girona and Dept of Biochemistry and Molecular Biology, Universidad de Girona, Girona; M. Tarón, Pangaea Biotech, USP Institut Universitari Dexeus, Barcelona and Dept of Medical Oncology, Catalan Institute of Oncology and Autonomous University of Barcelona, Hospital Germans Trias i Pujol, Barcelona; R. Colomer, Dept of Medical Oncology, M.D. Anderson España Cancer Center, Madrid; R. Rosell, Pangaea Biotech, USP Institut Universitari Dexeus, Barcelona and Dept of Medical Oncology, Catalan Institute of Oncology and Autonomous University of Barcelona, Hospital Germans Trias i Pujol, Barcelona, Spain.

The authors wish to thank the Spanish Lung Cancer Group (SLCG), sponsor of the SLADB study, and Roche Farma Spain, sponsor of the TargeT trial. We also acknowledge the investigators C. Santander, Dept of Medical Oncology, Hospital de San Millán, Logroño, Spain; P. Diz, Dept of Medical Oncology, Complejo Hospitalario de León, León, Spain; J. Oramas, Dept of Medical Oncology, Hospital Universitario de Canarias, Santa Cruz de Tenerife, Spain; R. Pérez, Dept of Medical Oncology, M.D. Anderson España Cancer Center, Madrid, Spain; A. García-Velasco, Dept of Medical Oncology, Catalan Institute of Oncology, Hospital Universitari Dr Josep Trueta, Girona, Spain; G. López, Dept of Medical Oncology, Hospital Universitario de Cruces, Baracaldo, Spain; A. Velasco, Dept of Medical Oncology, Hospital Universitario la Princesa, Madrid; S. del Barco, Dept of Medical Oncology, Catalan Institute of Oncology, Hospital Universitari Dr Josep Trueta; J. Montesinos, Dept of Medical Oncology, Fundacio Althaia-Xarxa Assistencial de Manresa, Manresa, Spain; P. Valero, Dept of Medical Oncology, Hospital Infanta Luisa, Seville, Spain; M. González, Hospital Universitario la Paz, Madrid, Spain; A. Montes, Dept of Medical Oncology, Catalan Institute of Oncology, L'Hospitalet, Spain; A. Izquierdo, Dept of Medical Oncology, Catalan Institute of Oncology, Hospital Universitari Dr Josep Trueta; J. Montalar, Dept of Medical Oncology, Hospital Universitario La Fe, Valencia, Spain; M. Amenedo, Dept of Medical Oncology, Centro Oncológico Regional de Galicia, La Coruña, Spain; C. Rolfo, Dept of Medical Oncology, Clínica Rotger, Palma de Mallorca, Spain; I. Bover, Dept of Medical Oncology, Hospital Son Llatzer, Mallorca, Spain; J.E. Alés, Dept of Medical Oncology, Complejo Hospitalario de Avila, Avila, Spain; P. Martínez, Dept of Medical Oncology, Hospital de Basurto, Bilbao, Spain; E. Felip, Dept of Medical Oncology, Hospital Universitari Vall d' Hebron, Barcelona, Spain; M. Cobo, Dept of Medical Oncology, Hospital Universitario Puerta de Hierro Majadahonda, Madrid; A.M. Jiménez, Dept of Medical Oncology, Hospital Universitario de Getafe, Madrid; M. Guillot, Dept of Medical Oncology, Hospital Son Dureta, Mallorca; A. Jaén, Dept of Medical Oncology, Complejo Hospitalario de Jaén, Jaén, Spain; P. Garrido, Dept of Medical Oncology, Hospital Universitario Ramón y Cajal, Madrid; C. Pallarès, Dept of Medical Oncology, Hospital Universitari de la Santa Creu i Sant Pau, Barcelona; and A. Cortijo, MD Anderson Cancer Center España, Madrid; as well as M.L. Amador and S. Figueroa, both Roche-Pharma, Basel, Switzerland; and J. Brunet, Dept of Medical Oncology, Catalan Institute of Oncology, Hospital Universitari Dr Josep Trueta. The authors also wish to thank all clinical research coordinators who collaborated in the analysis reported in this manuscript.

\section{REFERENCES}

1 Sorensen JB, Hansen $\mathrm{HH}$, Hansen $\mathrm{M}$, et al. Brain metastases in adenocarcinoma of the lung: frequency, risk groups, and prognosis. J Clin Oncol 1988; 6: 1474-1480.

2 Gaspar L, Scott C, Rotman M, et al. Recursive partitioning analysis (RPA) of prognostic factors in three Radiation Therapy Oncology Group (RTOG) brain metastases trials. Int J Radiat Oncol Biol Phys 1997; 37: 745-751.

3 Shepherd FA, Rodrigues Pereira J, Ciuleanu T, et al. Erlotinib in previously treated non-small-cell lung cancer. N Engl J Med 2005; 353: 123-132.

4 Lynch TJ, Bell DW, Sordella R, et al. Activating mutations in the epidermal growth factor receptor underlying responsiveness of nonsmall-cell lung cancer to gefitinib. N Engl J Med 2004; 350: 2129-2139.

5 Paez JG, Janne PA, Lee JC, et al. EGFR mutations in lung cancer: correlation with clinical response to gefitinib therapy. Science 2004; 304: 1497-1500.

6 Pao W, Miller V, Zakowski M, et al. EGF receptor gene mutations are common in lung cancers from "never smokers" and are associated with sensitivity of tumors to gefitinib and erlotinib. Proc Natl Acad Sci USA 2004; 101: 13306-13311.

$7 \mathrm{Ji} \mathrm{H}, \mathrm{Li} \mathrm{D}$, Chen L, et al. The impact of human EGFR kinase domain mutations on lung tumorigenesis and in vivo sensitivity to EGFRtargeted therapies. Cancer Cell 2006; 9: 485-495.

8 Politi K, Zakowski MF, Fan PD, et al. Lung adenocarcinomas induced in mice by mutant EGF receptors found in human lung cancers respond to a tyrosine kinase inhibitor or to downregulation of the receptors. Genes Dev 2006; 20: 1496-1510.

9 Shigematsu H, Lin L, Takahashi T, et al. Clinical and biological features associated with epidermal growth factor receptor gene mutations in lung cancers. J Natl Cancer Inst 2005; 97: 339-346.

10 Rosell R, Taron M, Reguart N, et al. Epidermal growth factor receptor activation: how exon 19 and 21 mutations changed our understanding of the pathway. Clin Cancer Res 2006; 12: 7222-7231.

11 Rosell R, Moran T, Queralt C, et al. Screening for epidermal growth factor receptor mutations in lung cancer. N Engl J Med 2009; 361: 958-967.

12 Therasse P, Arbuck SG, Eisenhauer EA, et al. New guidelines to evaluate the response to treatment in solid tumors. European Organization for Research and Treatment of Cancer, National Cancer Institute of the United States, National Cancer Institute of Canada. J Natl Cancer Inst 2000; 92: 205-216.

13 Common Terminology Criteria for Adverse Events version 3.0 www.ctep.cancer.gov/reporting/ctc Date last accessed January 12, 2011. Date last updated September 7, 2010.

14 Jackman DM, Miller VA, Cioffredi LA, et al. Impact of epidermal growth factor receptor and KRAS mutations on clinical outcomes in previously untreated non-small cell lung cancer patients: results of an online tumor registry of clinical trials. Clin Cancer Res 2009; 15: $5267-5273$. 
15 Serizawa T. Radiosurgery for metastatic brain tumors. Int J Clin Oncol 2009; 14: 289-298.

16 Das AK, Sato M, Story MD, et al. Non-small-cell lung cancers with kinase domain mutations in the epidermal growth factor receptor are sensitive to ionizing radiation. Cancer Res 2006; 66: 9601-9608.

17 Gow CH, Chien CR, Chang YL, et al. Radiotherapy in lung adenocarcinoma with brain metastases: effects of activating epidermal growth factor receptor mutations on clinical response. Clin Cancer Res 2008; 14: 162-168.

18 Adamo V, Franchina T, Adamo B, et al. Brain metastases in patients with non-small cell lung cancer: focus on the role of chemotherapy. Ann Oncol 2006; 17: Suppl. 2, ii73-75.

19 Colomer R, Cosos D, Del Campo JM, et al. Brain metastases from breast cancer may respond to endocrine therapy. Breast Cancer Res Treat 1988; 12: 83-86.

20 Cappuzzo F, Ardizzoni A, Soto-Parra H, et al. Epidermal growth factor receptor targeted therapy by ZD 1839 (Iressa) in patients with brain metastases from non-small cell lung cancer (NSCLC). Lung Cancer 2003; 41: 227-231.

21 Namba Y, Kijima T, Yokota S, et al. Gefitinib in patients with brain metastases from non-small-cell lung cancer: review of 15 clinical cases. Clin Lung Cancer 2004; 6: 123-128.
22 Poon AN, Ho SS, Yeo W, et al. Brain metastasis responding to gefitinib alone. Oncology 2004; 67: 174-178.

23 Takahashi H, Ohrui T, Ebihara S, et al. Effect of gefitinib (ZD1839) on metastatic brain tumour. Lung Cancer 2004; 43: 371-372.

24 Ceresoli GL, Cappuzzo F, Gregorc V, et al. Gefitinib in patients with brain metastases from non-small-cell lung cancer: a prospective trial. Ann Oncol 2004; 15: 1042-1047.

25 Omuro AM, Kris MG, Miller VA, et al. High incidence of disease recurrence in the brain and leptomeninges in patients with nonsmall cell lung carcinoma after response to gefitinib. Cancer 2005; 103: 2344-2348.

26 Yi HG, Kim HJ, Kim YJ, et al. Epidermal growth factor receptor (EGFR) tyrosine kinase inhibitors (TKIs) are effective for leptomeningeal metastasis from non-small cell lung cancer patients with sensitive EGFR mutation or other predictive factors of good response for EGFR TKI. Lung Cancer 2009; 65: 80-84.

27 Katayama T, Shimizu J, Suda K, et al. Efficacy of erlotinib for brain and leptomeningeal metastases in patients with lung adenocarcinoma who showed initial good response to gefitinib. J Thorac Oncol 2009; 4: 1415-1419.

28 Perez-Soler R, Chachoua A, Hammond LA, et al. Determinants of tumor response and survival with erlotinib in patients with nonsmall-cell lung cancer. J Clin Oncol 2004; 22: 3238-3247. 